Mise au point

\title{
La prévention des blessures sportives : modèles théoriques et éléments-clés d'une stratégie efficace
}

\section{The prevention of sports injuries: Conceptual models and keys elements of an efficient strategy}

\author{
F. Delvaux ${ }^{\mathrm{a}, *}$, J.-F. Kaux ${ }^{\mathrm{b}}$, B. Forthomme ${ }^{\mathrm{a}}$, J.-L. Croisier ${ }^{\mathrm{a}}$ \\ a Université de Liège, ISEPK, B21, allée des Sports 4, 4000 Liège, Belgique \\ b $\mathrm{CHU}$ de Liège, avenue de l'Hôpital, 1, 4000 Liège, Belgique
}

\section{N F O A R T I C L E}

\section{Historique de l'article :}

Disponible sur Internet le 25 août 2018

\section{Mots clés :}

Prévention lésionnelle

Modèle théorique

Facteurs de risque

Stratégie préventive

\begin{abstract}
R É S U M É
La recherche sur la thématique de la prévention lésionnelle a connu ces dernières années, et toujours actuellement, un développement important. Plusieurs modèles théoriques ont été décrits afin d'optimiser l'efficacité des stratégies préventives qui, si elles sont créées et pratiquées de manière rigoureuse, peuvent s'avérer particulièrement efficaces. Parmi ces modèles, nous pouvons notamment pointer les très récents " sports injury risk management approaches " qui pourraient s'avérer pertinents dans le futur mais qui doivent être confirmés par des études. Pour être efficace, une stratégie préventive devrait notamment inclure divers éléments tels que : un bilan médico-sportif de pré-saison, une action coordonnée sur plusieurs facteurs de risque, une compliance maximale des participants ainsi que du staff, et une quantification/gestion de la charge de travail.

(C) 2018 Publié par Elsevier Masson SAS.
\end{abstract}

Keywords:

Injury prevention

Theoretical model

Risk factors

Preventive strategies

\section{A B S T R A C T}

Research topic about sport injury prevention has importantly increased these last years. Several theoretical models have been developed with the aim of helping sport and medical staffs with preventive strategies, and many studies have demonstrated the high efficacy of preventive programs if quality conditions are met. Among these models, at least two "sports injury risk management approaches" have been recently described and their relevance needs to be confirmed by further research. In order to maximize efficacy, preventive strategies should include: pre-competition medical assessment, actions to decrease multiple risk factors at the same time, maximal compliance, training load quantification and monitoring. (C) 2018 Published by Elsevier Masson SAS.

\section{Introduction}

La prévention de blessures liées à la pratique sportive est devenue une préoccupation majeure dans le domaine des sciences et de la médecine du sport. Le nombre d'études sur cette thématique augmente chaque année depuis 1990, et cette croissance s'est encore fortement accentuée depuis 2010. La multiplication d'essais contrôlés randomisés a permis désormais d'alimenter des revues systématiques et/ou méta-analyses, et ainsi d'optimiser les

\footnotetext{
* Auteur correspondant.

Adresse e-mail : fdelvaux@uliege.be (F. Delvaux).
}

recommandations sur la mise en place de stratégies de prévention lésionnelle [1]. Nous possédons également de meilleures connaissances sur l'impact que peut avoir une blessure sur la performance, notamment en sports collectifs. À titre d'exemple, dans une vaste étude d'une durée de 11 ans centrée sur le football professionnel européen, Hägglund et al. ont mis en évidence une relation claire entre une faible incidence lésionnelle et la performance du club tant dans le championnat domestique que dans les compétitions européennes [2] ; ce constat a également été observé dans le football professionnel français et qatari $[3,4]$. À côté de ce coût "sportif ", la survenue de blessures peut aussi représenter un coût financier très élevé, d'autant plus si la blessure survient chez un athlète évoluant dans une structure de haut niveau. Ainsi, le coût moyen 
Tableau 1

Modèles de van Mechelen et al. [12] et Translating Research Into Prevention Practice (TRIPP) [13].

\begin{tabular}{|c|c|c|}
\hline Étape du modèle & van Mechelen et al. & TRIPP \\
\hline 1 & Décrire l'étendue du problème (recenser incidence et sévérité) & Idem \\
\hline 2 & Identifier les facteurs de risque et mécanismes lésionnels & Idem \\
\hline 3 & Mettre en place les mesures préventives & Développer les mesures préventives \\
\hline 4 & Évaluer l'efficacité des mesures préventives (répéter l'étape 1) & Évaluer l'efficacité des mesures dans des conditions idéales \\
\hline 5 & - & Décrire le contexte réel d'intervention pour favoriser son implémentation \\
\hline 6 & - & Évaluer l'efficacité des mesures dans le contexte réel \\
\hline
\end{tabular}

associé à une blessure d'un footballeur professionnel d'un club du top européen serait estimé à 500000 euros pour un mois d'absence [5] ; en National Football League (football américain), un préjudice d'environ 350000 US Dollars par club et par saison serait uniquement lié à la survenue de lésions musculaires des ischio-jambiers au sein de l'équipe [6].

Une recherche en plein essor, un coût sportif et financier lié à des blessures parfois exorbitant ne semblent cependant pas suffire à produire des résultats que nous pourrions qualifier de satisfaisants. Certes, l'efficacité spectaculaire de certains programmes préventifs comme le Nordic Hamstrings [7] ou le Copenhagen Adductors $[8,9]$ n'est plus à démontrer. Cependant, si Ekstrand et al. [10] ont observé une baisse significative de l'incidence globale de blessures ligamentaires dans le football européen entre 2001 et 2012, aucune réduction de cette incidence n'a pu être mise en évidence pour ce qui concerne les lésions musculaires ainsi que les lésions qualifiées de sévères (c'est-à-dire causant une absence de participation aux entraînements et aux matchs de plus de 28 jours). Il apparaît donc que nos efforts destinés à empêcher la survenue de blessures liées à la pratique d'un sport sont encourageants mais que nous sommes loin d'avoir atteint une efficacité optimale. De plus, dans un exercice certes périlleux mais néanmoins intéressant, Waldén [11] a extrapolé, sur la base des tendances observées dans l'étude d'Ekstrand [10], qu'une équipe professionnelle de football composée de 25 joueurs pouvait s'attendre, en 2032, à 4 blessures de hanche/pubalgie, 12 lésions musculaires des ischio-jambiers, 2 à 3 entorses de cheville et 2 à 3 ruptures du ligament croisé antérieur par saison... Ces chiffres, a priori alarmants, sont donc purement hypothétiques, mais plaident pour une réflexion profonde au développement d'approches préventives de qualité.

L'objectif de cet article est double :

- faire le point sur les modèles et fondements théoriques utilisés en prévention lésionnelle ;

- recenser les éléments essentiels composant une stratégie préventive efficace.

\section{D'un modèle à un autre ?}

Dans l'optique de maximiser l'efficacité de stratégies préventives, certains auteurs ont développé des modèles théoriques de prévention lésionnelle destinés aux acteurs de terrain. Le plus connu d'entre eux est probablement Willem van Mechelen qui, dès 1992, a adapté un modèle de prévention de santé publique au contexte des lésions sportives [12]. La première étape de ce modèle, qui en comporte quatre, consiste à recenser les données épidémiologiques telles que l'incidence et la sévérité des blessures au sein d'une population cible ; en deuxième lieu, il s'agit d'identifier et de comprendre les facteurs de risque et les mécanismes lésionnels de la pathologie en question ; la troisième étape consiste en la mise en place effective des mesures préventives, et la quatrième étape nécessite une mesure de l'efficacité des mesures préventives introduites en répétant l'étape 1. Quelques années plus tard, estimant notamment que ce modèle ne permettait pas une implémentation satisfaisante sur le terrain,
Finch [13] a proposé le modèle Translation Research into Injury Prevention Practice (TRIPP). Si les deux premières étapes se distinguent peu du modèle de van Mechelen, Finch recommande encore quatre étapes supplémentaires comme décrit dans le Tableau 1.

Plus récemment, plusieurs spécialistes en prévention lésionnelle ont remis en question ces modèles, en visant plus particulièrement leur caractère non dynamique [14] ou surtout la pertinence des outils utilisés pour prédire une future blessure. Bahr [15] estime notamment que pour obtenir d'un test une valeur prédictive convaincante de future blessure, celui-ci devrait répondre à trois critères successifs :

- une forte corrélation doit être établie entre un marqueur de ce test et le risque lésionnel ;

- l'usage de ce test doit être répété parmi une population spécifique en séparant, selon des valeurs-seuil prédéterminées, les athlètes à risque élevé de blessure des autres ;

- l'intervention, sous forme d'essai contrôlé randomisé, doit démontrer un bénéfice plus grand parmi le groupe de sujets à risque élevé que parmi la population contrôle.

Ainsi, l'auteur conclut sans équivoque qu'à ce jour, il n'existe aucun outil validé sur la base de ces trois étapes et donc qu'aucun test n'est actuellement capable de prédire une future blessure... et que ce ne sera probablement jamais le cas ! Van Dyk et Clarsen [16] abondent dans ce sens et rappellent que le fait d'associer un facteur de risque avec une blessure ne signifie pas que nous pouvons identifier précisément des athlètes à risque, c'est-à-dire que ce facteur de risque ne pourra pas représenter un élément prédictif suffisamment fiable de lésion ultérieure. Ils exhortent les scientifiques à développer plutôt des stratégies préventives faciles à implémenter sur le terrain, comme par exemple le programme FIFA $11+$, et à observer son efficacité parmi différentes populations spécifiques.

Bittencourt et al. considèrent que le modèle de van Mechelen est surtout un modèle linéaire qui ne peut donc prendre en compte les interactions entre les différents facteurs de risque lésionnels [17]. Ils recommandent plutôt d'analyser la nature complexe et multifactorielle d'une blessure sportive via une toile de déterminants de cette blessure. En d'autres mots, ces auteurs préconisent de réfléchir à la manière dont les facteurs de risque interagissent entre eux et d'identifier ainsi des profils à risque ( injury pattern recognition ») plutôt que de compiler des facteurs de risque en unités séparées. La Fig. 1 illustre cette toile de déterminants d'une rupture du ligament croisé antérieur (LCA) dans deux contextes : la première dans le basketball (Fig. 1A) et la seconde dans le milieu de la danse classique (Fig. 1B), avec des différences notoires en fonction du contexte sportif.

Arguant que prévenir la survenue de blessure était impossible et qu'il valait mieux se fixer l'objectif de réduire au minimum le risque lésionnel, une équipe irlandaise préconise de se baser sur une stratégie de gestion de risque qui garantirait une faible susceptibilité aux blessures [18]. Les auteurs recommandent ainsi aux acteurs de terrain, pour réaliser une 

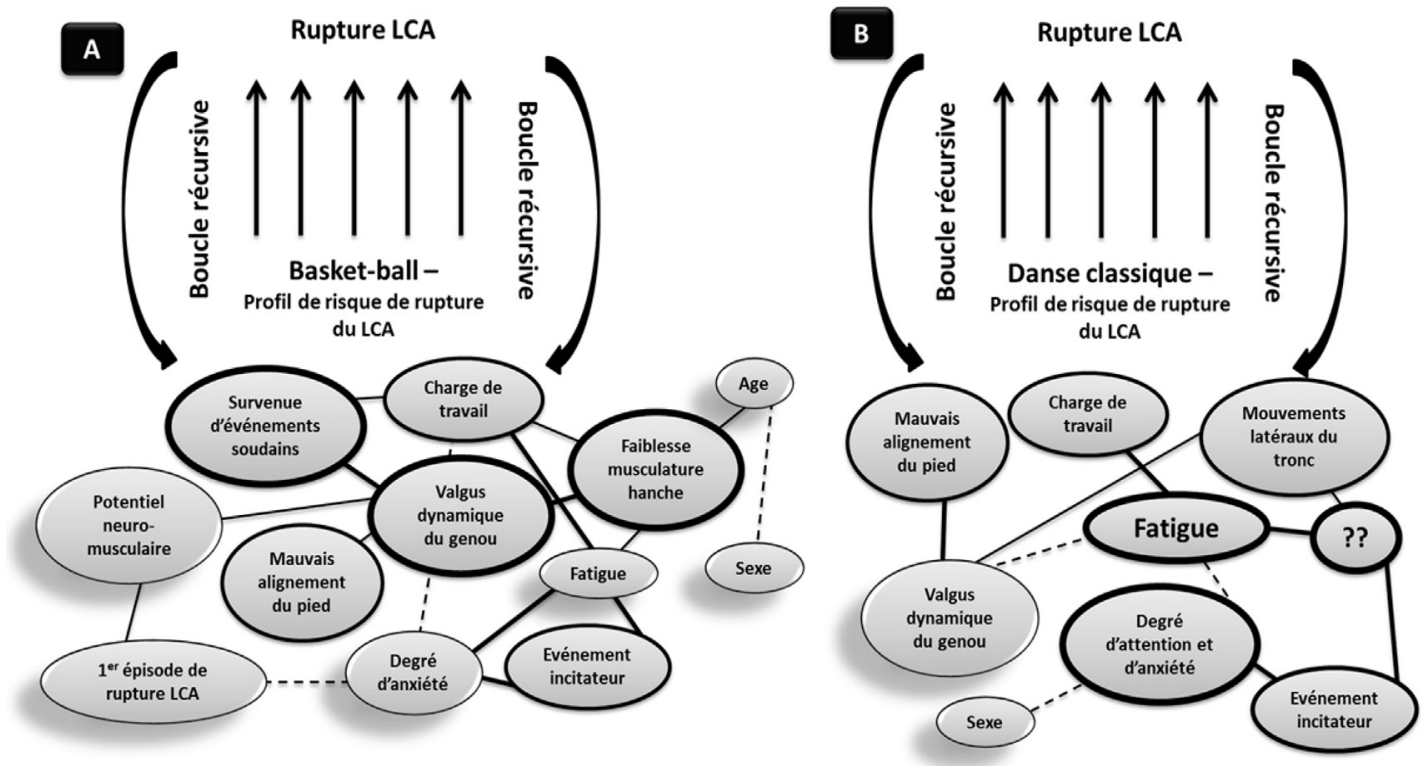

Fig. 1. Toile de déterminants d'une rupture du LCA : chez un athlète pratiquant le basketball (A); chez un athlète pratiquant la danse classique (B) (d'après Bittencourt et al.) [17].

démarche préventive efficace et basée sur l'évidence scientifique, de suivre les six étapes de ce modèle (" Operational framework for individualising injury risk management in sport " ; Fig. 2) :

- recenser quand, où et comment des athlètes peuvent développer certaines blessures ;
- identifier les éléments qui augmentent ou réduisent le risque lésionnel :

- comprendre les demandes de la discipline sportive en question : pour quels types d'efforts l'athlète doit-il être préparé ? ;

- connaître le profil de l'athlète : présente-t-il des caractéristiques de susceptibilité ou de protection vis-à-vis de certaines blessures? ;

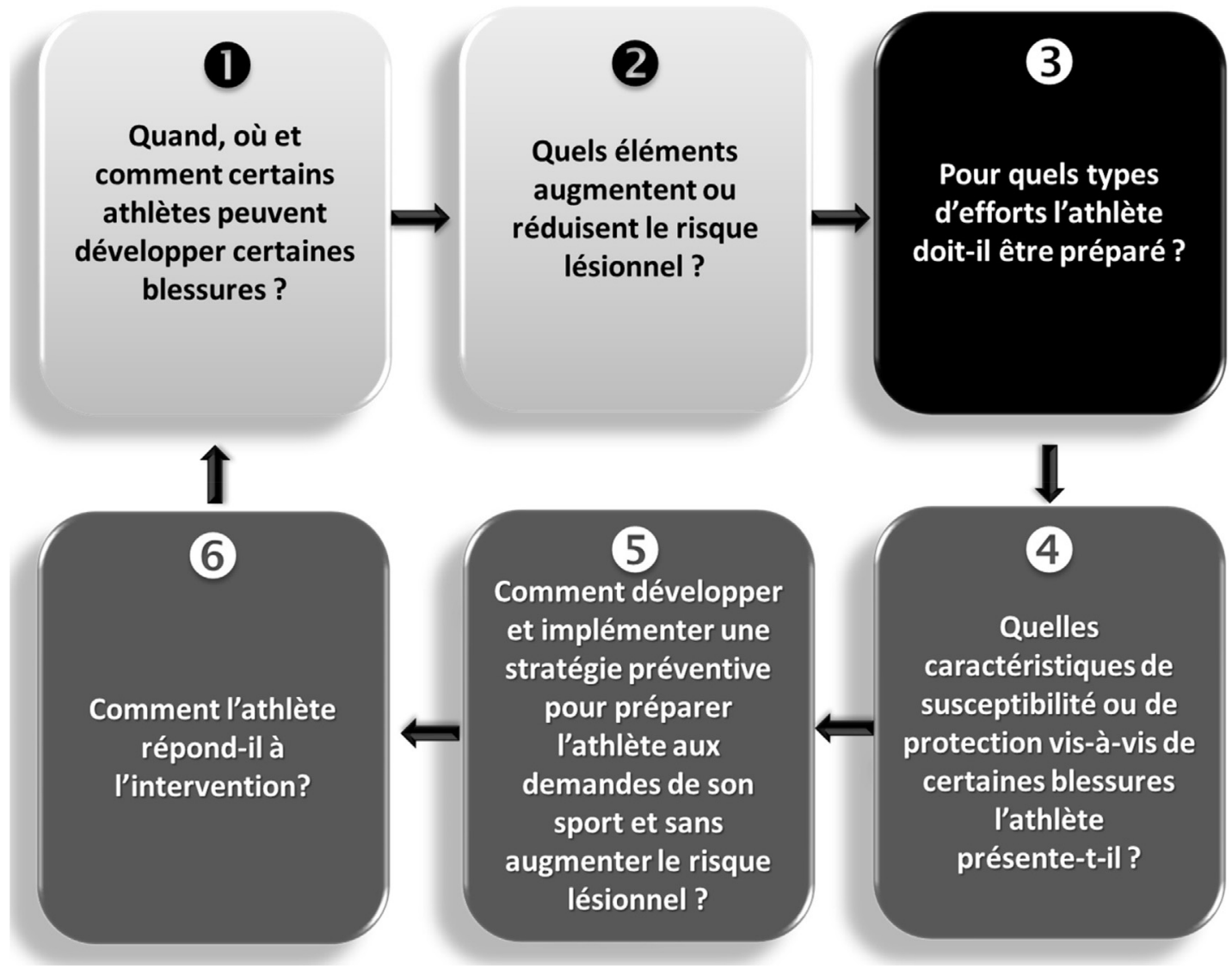

Fig. 2. "Operational framework for indivualizing injury risk management in sport " (d'après de Roe et al.) [18]. 


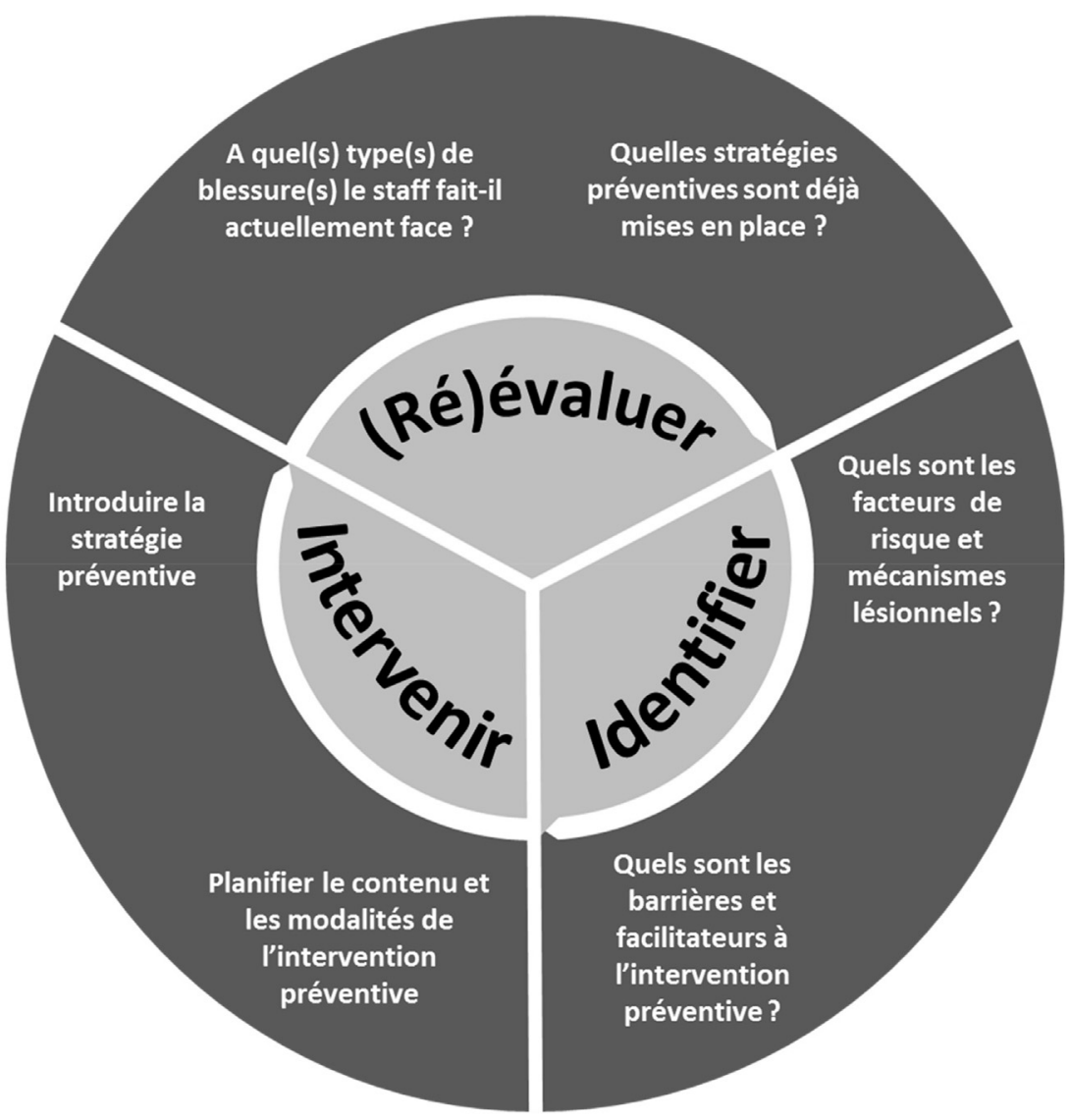

Fig. 3. Le « Team-Sport Injury Prevention (TIP) Cycle » (d'après Pruna et al., 2018) [19].

- développer une stratégie interventionnelle pour préparer l'athlète aux demandes de son sport sans augmenter le risque lésionnel ;

- mettre en place un suivi de l'athlète : comment celui-ci répond-il à la charge imposée?

Ce type de stratégie que l'on pourrait qualifier de risk management approach est également à l'origine d'un nouveau modèle développé par le FC Barcelone en collaboration avec le Oslo Sports Trauma Research Center et destiné surtout aux sports collectifs [19]. Le team-sport injury prevention (TIP) se compose d'un cycle permanent basé sur trois phases-clés (Fig. 3). Durant la phase 1 ou phase d'évaluation, deux questions sont posées :

- à quel(s) type(s) de blessure(s) le staff fait-il face actuellement ? - quelles stratégies préventives sont mises en place pour éviter cette/ces blessure(s)?

La phase suivante (phase 2 - identification) requiert une analyse des facteurs de risque et des mécanismes lésionnels, et s'accompagne d'une analyse des éléments représentant une barrière ou à caractère plutôt facilitateur lors de la mise en place d'une stratégie préventive. Enfin, la troisième phase (phase d'intervention) nécessite de planifier tant le contenu (ce qui doit être activé) que la forme (comment et par qui l'action sera menée) de l'intervention préventive, et de mettre en place celle-ci.

Étant donné le nombre de modèles et les critiques que l'on pourrait formuler à l'encontre de chacun d'entre eux, nous suggérons aux praticiens de s'approprier celui qui semble convenir le mieux à l'ensemble des membres du staff médico-sportif. Une fois le modèle adopté, le suivi rigoureux de chaque étape apparaît primordial si l'on souhaite assurer une efficacité optimale à la stratégie préventive.

\section{Quels éléments-clés pour optimiser l'efficacité de la stratégie préventive?}

Le contenu de la stratégie préventive dépend évidemment de nombreux paramètres : le(s) type(s) de blessures que l'on souhaite éviter prioritairement, les possibilités pratiques d'implémenter l'intervention, le coût humain et financier, les spécificités de la population en question (âge, sexe, niveau de jeu, ...), etc. Cependant, malgré les différences que l'on observe parmi la multitude de ces programmes préventifs, nous pouvons identifier un certain nombre de points qui devraient être inclus dans une stratégie préventive efficace.

\subsection{Un bilan médical de pré-saison}

Utile pour détecter des éléments qui pourraient prédisposer au développement de futures pathologies, le bilan médical devrait d'abord établir les antécédents médicaux de l'athlète. Il est, en effet, communément admis que la présence d'un antécédent lésionnel représente un facteur de risque majeur d'une récidive [20] pour de multiples raisons, notamment une récupération incomplète depuis la dernière blessure [21]. Certaines anomalies, symptomatiques ou non, peuvent permettre de diriger l'athlète vers un programme de remédiation ou de rééducation. À titre d'exemple, la présence d'une dyskinésie scapulaire favoriserait la survenue de blessure à l'épaule [22] ; la présence d'une instabilité de cheville favoriserait la survenue ultérieure d'entorse externe de cette même cheville [23] ; un faible contrôle moteur lombo-pelvien majorerait le risque de 
développer un épisode de lombalgie [24] ; une faiblesse musculaire des ischio-jambiers augmenterait la propension à développer une lésion musculaire des mêmes muscles [25]. Tout élément susceptible de jouer un rôle dans la survenue d'une lésion ultérieure devrait être pris en compte lors de cet examen, et cela peut notamment inclure l'utilisation de technologies pointues (analyses vidéos ou 3D, isocinétisme, imagerie,...) en marge de l'examen médical classique.

\subsection{Une action coordonnée sur plusieurs facteurs de risque}

Ces dernières années, un certain nombre de programmes préventifs ont vu le jour comme le Prevent Injury and Enhance Performance (PEP) ou le FIFA 11+, et ont démontré leur efficacité parmi différentes populations [26-28]. Une des raisons de leur succès est due au fait que ces programmes contiennent une variété d'exercices dont l'objectif est d'améliorer force, proprioception, agilité, plyométrie, qualité de gestuelle ou encore souplesse : ainsi, en un temps assez réduit (moins de 30 minutes par session), un groupe d'athlètes pourra améliorer l'ensemble de différentes qualités entraînables qui sont liées de près ou de loin à un risque lésionnel (si faiblesse de celles-ci). Leur simplicité, leur faible coût, leur facilité de compréhension et de possibilités pratiques d'implémentation (pas de nécessité de matériel particulier) représentent aussi des atouts majeurs de ces programmes. Il est intéressant de noter que le programme FIFA 11+ s'est avéré efficace dans d'autres sports que le football, en l'occurrence le basketball [29]. Ce type de programme pourrait donc s'appliquer à d'autres contextes que le football, à la condition probable d'adapter certaines composantes à la spécificité de la discipline en question.

\subsection{Une compliance maximale}

Plusieurs études ont identifié une relation inverse entre la compliance à un programme préventif et le risque lésionnel $[27,30]$. Cela signifie que plus le programme préventif est réalisé régulièrement, plus le risque de se blesser diminue. Parmi une large cohorte de footballeurs, Silvers-Granelli et al. ont objectivé l'importance de la compliance au programme FIFA 11+ sur le risque lésionnel global et le nombre de journées d'absence des terrains pour cause de blessures [27]. Des résultats abondant dans ce sens avaient également été retrouvés chez de jeunes footballeuses : la compliance de ces athlètes était en lien avec une réduction d'incidence de rupture du LCA ainsi que de lésions aiguës au genou [30]. Une revue systématique avec méta-analyse récente a conclu qu'un programme de renforcement des ischio-jambiers en mode excentrique était efficace ( $65 \%$ de réduction de risque lésionnel) mais à la condition fondamentale que la compliance des participants soit maximale [31]. De plus, pour optimiser la compliance et donc l'efficacité du programme, il apparaît indispensable que tous les membres du staff médico-sportif l'adoptent et que ce programme soit réaliste et adapté à la population ciblée [32].

\subsection{Une quantification et une gestion de la charge de travail}

La charge de travail est considérée comme un facteur de risque important de blessure [33], tant pour les blessures microtraumatiques que macrotraumatiques. Le Comité Olympique International, dans une déclaration de consensus datant de 2016 et à but préventif, recommande d'ailleurs de mesurer chez tous les athlètes la charge de travail $[34,35]$. La manière d'évaluer cette charge peut varier d'une situation à une autre, mais l'outil le plus fréquemment utilisé est le Rating of Perceived Exertion (RPE) de Foster [36], qui correspond au produit de la durée d'effort (en minutes) par l'intensité ressentie par l'athlète (sur une échelle de 0 à 10) pour chaque session sportive. Certains auteurs, parmi des populations issues de différents sports, ont identifié des normes en terme d'unités arbitraires (UA) hebdomadaires absolues qui étaient associées à un risque accru de blessures ou à un risque protecteur [37-39]. À ce jour, d'autres études semblent nécessaires pour obtenir des données plus consistantes en terme d'UA à ne pas dépasser (ou à dépasser en cas de manque de préparation), mais il apparaît nécessaire de considérer les variations de charge de travail, mesurées via un ratio charge aiguë/charge chronique [40]. McCall et al. ont par exemple démontré que ces ratios mesurés sur trois ou quatre semaines étaient associés à un risque majoré de blessures dans le football professionnel [33]. Cependant, malgré ces résultats intéressants, aucun pouvoir prédictif de future lésion lié à ce ratio n'a pu être établi : la prédiction d'une blessure sportive ne pourra définitivement jamais être considérée comme une science exacte [41].

\section{Conclusion}

La recherche sur la thématique de la prévention lésionnelle est actuellement en plein essor ; plusieurs modèles théoriques ont été décrits afin d'optimiser l'efficacité des stratégies préventives qui, si elles sont développées et pratiquées de manière rigoureuse, peuvent s'avérer très efficaces. Parmi ces modèles, nous pouvons notamment pointer les " sports injury risk management approaches " qui pourraient s'avérer pertinents dans le futur mais qui doivent être confirmés par des études. Enfin, pour être efficace, une stratégie préventive devrait notamment inclure un bilan médico-sportif de pré-saison, une action coordonnée sur plusieurs facteurs de risque, une compliance maximale des participants et du staff, ainsi qu'une quantification et une gestion de la charge de travail.

\section{Remerciements}

Nous remercions chaleureusement Madame Annie Depaifve pour ses relectures attentives et son aide précieuse à l'élaboration des figures et tableaux.

\section{Déclaration de liens d'intérêts}

Les auteurs déclarent ne pas avoir de liens d'intérêts.

\section{Références}

[1] Vriend I, Gouttebarge V, Finch CF, van Mechelen W, Verhagen EALM. Intervention strategies used in sport injury prevention studies: a systematic review identifying studies applying the Haddon Matrix. Sports Med 2017;47:2027-43.

[2] Hagglund M, Walden M, Magnusson H, Kristenson K, Bengtsson H, Ekstrand J. Injuries affect team performance negatively in professional football: an 11year follow-up of the UEFA Champions League injury study. Br J Sports Med 2013; $47: 738-42$

[3] Carling C, Le Gall F, McCall A, Nedelec M, Dupont G. Squad management, injury and match performance in a professional soccer team over a championshipwinning season. Eur J Sport Sci 2015;15:573-82.

[4] Eirale C, Tol JL, Faroog A, Smiley F, Chalabi H. Low injury rate strongly correlates with team success in Qatari professional football. Br J Sports Med 2013;47:807-8

[5] Ekstrand J, Hagglund M, Walden M. Injury incidence and injury patterns in professional football: the UEFA injury study. Br J Sports Med 2011;45(7):553-8.

[6] Elliott MCCW, Zarins B, Powell JW, Kenyon CD. Hamstring muscle strains in professional football players: a 10-year review. Am J Sports Med 2011;39:843-50.

[7] van der Horst N, Smits D-W, Petersen J, Goedhart EA, Backx FJG. The preventive effect of the nordic hamstring exercise on hamstring injuries in amateur soccer players: a randomized controlled trial. Am J Sports Med 2015;43:1316-23.

[8] Haroy J, Clarsen B, Wiger EG, Oyen MG, Serner A, Thorborg K, et al. The Adductor Strengthening Programme prevents groin problems among male football players: a cluster-randomised controlled trial. Br J Sports Med 2018;0:1-8.

[9] Haroy J, Thorborg K, Serner A, Bjorkheim A, Rolstad LE, Holmich P, et al. Including the Copenhagen adduction exercise in the FIFA 11+ provides missing eccentric hip adduction strength effect in male soccer players: a randomized controlled trial. Am J Sports Med 2017;45:3052-9.

[10] Ekstrand J, Hagglund M, Kristenson K, Magnusson H, Walden M. Fewer ligament injuries but no preventive effect on muscle injuries and severe injuries: an 11 year follow-up of the UEFA Champions League injury study. Br J Sports Med 2013;47:732-7. 
[11] Waldén M. Reflecting on the data patterns of 15 years: a future prediction for 2032. In: The future of football medicine. Calzetti Mariucci Editori; 2017. p. 21 [International Conference on Sports Rehabilitation and Traumatology].

[12] van Mechelen W, Hlobil H, Kemper HC. Incidence, severity, aetiology and prevention of sports injuries. A review of concepts. Sports Med 1992;14: 82-99.

[13] Finch C. A new framework for research leading to sports injury prevention. J Sci Med Sport 2006;9:3-9 [discussion 10].

[14] Cook C. Predicting future physical injury in sports: it's a complicated dynamic system. Br J Sports Med 2016;50:1356-7.

[15] Bahr R. Why screening tests to predict injury do not work-and probably never will.: a critical review. Br J Sports Med 2016;50:776-80.

[16] van Dyk N, Clarsen B. Prevention forecast: cloudy with a chance of injury. Br J Sports Med 2017;51:1646-7.

[17] Bittencourt NFN, Meeuwisse WH, Mendonca LD, Nettel-Aguirre A, Ocarino JM Fonseca ST. Complex systems approach for sports injuries: moving from risk factor identification to injury pattern recognition-narrative review and new concept. Br J Sports Med 2016;50(21):1309-14.

[18] Roe M, Malone S, Blake C, Collins K, Gissane C, Buttner F, et al. A six stage operational framework for individualising injury risk management in sport. Inj Epidemiol 2017:4:26.

[19] Pruna R, Andersen TE, Clarsen B, McCall A. Muscle injury guide: prevention and return to play from muscle injuries. Barça Innovation Hub; 2018.

[20] Hagglund M, Walden M, Ekstrand J. Previous injury as a risk factor for injury in elite football: a prospective study over two consecutive seasons. Br J Sports Med 2006;40:767-72.

[21] Croisier J-L, Forthomme B, Namurois M-H, Vanderthommen M, Crielaard J-M. Hamstring muscle strain recurrence and strength performance disorders. Am J Sports Med 2002;30:199-203.

[22] Clarsen B, Bahr R, Andersson SH, Munk R, Myklebust G. Reduced glenohumera rotation, external rotation weakness and scapular dyskinesis are risk factors for shoulder injuries among elite male handball players: a prospective cohort study. Br J Sports Med 2014;48:1327-33.

[23] Pourkazemi F, Hiller CE, Raymond J, Nightingale EJ, Refshauge KM. Predictors of chronic ankle instability after an index lateral ankle sprain: a systematic review. J Sci Med Sport 2014;17:568-73.

[24] Cholewicki J, Silfies SP, Shah RA, Greene HS, Reeves NP, Alvi K, et al. Delayed trunk muscle reflex responses increase the risk of low back injuries. Spine 2005;30:2614-20.

[25] Croisier J-L, Ganteaume S, Binet J, Genty M, Ferret J-M. Strength imbalances and prevention of hamstring injury in professional soccer players: a prospective study. Am J Sports Med 2008;36:1469-75

[26] Bizzini M, Dvorak J. FIFA 11+: an effective programme to prevent football injuries in various player groups worldwide - A narrative review. Br J Sports Med 2015;49:577-9.

[27] Silvers-Granelli HJ, Bizzini M, Arundale A, Mandelbaum BR, Snyder-Mackler L Higher compliance to a neuromuscular injury prevention program improves overall injury rate in male football players. Knee Surg Sports Traumatol Arthrosc 2018;26:1975-83, http://dx.doi.org/10.1007/s00167-018-4895-5.

[28] Silvers-Granelli H, Mandelbaum B, Adeniji O, Insler S, Bizzini M, Pohlig R, et al. Efficacy of the FIFA 11+ injury prevention program in the collegiate male soccer player. Am J Sports Med 2015;43:2628-37.

[29] Longo UG, Loppini M, Berton A, Marinozzi A, Maffulli N, Denaro V. The FIFA $11+$ program is effective in preventing injuries in elite male basketball players: a cluster randomized controlled trial. Am J Sports Med 2012;40: 996-1005.

[30] Hagglund M, Atroshi I, Wagner P. Walden M. Superior compliance with a neuromuscular training programme is associated with fewer ACL injuries and fewer acute knee injuries in female adolescent football players: secondary analysis of an RCT. Br J Sports Med 2013;47:974-9.

[31] Goode AP, Reiman MP, Harris L, DeLisa L, Kauffman A, Beltramo D, et al. Eccentric training for prevention of hamstring injuries may depend on intervention compliance: a systematic review and meta-analysis. Br J Sports Med 2015;49:349-56

[32] Donaldson A, Callaghan A, Bizzini M, Jowett A, Keyzer P, Nicholson M. A concept mapping approach to identifying the barriers to implementing an evidencebased sports injury prevention programme. Inj Prev J Int Soc Child Adolesc Inj Prev 2017:23:273-80.

[33] McCall A, Dupont G, Ekstrand J. Internal workload and non-contact injury: a one-season study of five teams from the UEFA Elite Club Injury Study. Br Sports Med 2018:0:1-6.

[34] Soligard T, Schwellnus M, Alonso J-M, Bahr R, Clarsen B, Dijkstra HP, et al. How much is too much? (Part 1) International Olympic Committee consensus statement on load in sport and risk of injury. Br J Sports Med 2016:50:1030-41.

[35] Schwellnus M, Soligard T, Alonso J-M, Bahr R, Clarsen B, Dijkstra HP, et al. How much is too much? (Part 2) International Olympic Committee consensus statement on load in sport and risk of illness. Br J Sports Med 2016;50:1043-52.

[36] Foster C, Florhaug JA, Franklin J, Gottschall L, Hrovatin LA, Parker S, et al. A new approach to monitoring exercise training. J Strength Cond Res 2001;15:109-15.

[37] Malone S, Owen A, Newton M, Mendes B, Collins KD, Gabbett TJ. The acute: chonic workload ratio in relation to injury risk in professional soccer. J Sci Med Sport 2017:20:561-5.

[38] Windt J, Gabbett TJ, Ferris D, Khan KM. Training load-injury paradox: is greater preseason participation associated with lower in-season injury risk in elite rugby league players? Br J Sports Med 2017;51:645-50.

[39] Stares J, Dawson B, Peeling P, Heasman J, Rogalski B, Drew M, et al. Identifying high risk loading conditions for in-season injury in elite Australian footbal players. J Sci Med Sport 2018;21:46-51.

[40] Blanch P, Gabbett T]. Has the athlete trained enough to return to play safely? The acute: chronic workload ratio permits clinicians to quantify a player's risk of subsequent injury. Br J Sports Med 2016;50:471-5.

[41] Hulin BT, Gabbett TJ. Indeed association does not equal prediction: the neverending search for the perfect acute: chronic workload ratio. Br J Sports Med 2018;0:1-2. 